\title{
Role of monocyte-to-lymphocyte ratio in predicting sorafenib response in patients with advanced hepatocellular carcinoma
}

This article was published in the following Dove Press journal:

OncoTargets and Therapy

\author{
Zhenfeng Zhu' ${ }^{1,2, *}$ \\ Litao $\mathrm{Xu}^{1,2, *}$ \\ Liping Zhuang ${ }^{1,2}$ \\ Zhouyu Ning ${ }^{1,2}$ \\ Chenyue Zhang ${ }^{1,2}$ \\ Xia Yan ${ }^{1,2}$ \\ Junhua $\operatorname{Lin}^{1,2}$ \\ Yehua Shen ${ }^{1,2}$ \\ Peng Wang ${ }^{1,2}$ \\ Zhiqiang Meng ${ }^{1,2}$
}

'Department of Integrative Oncology, Fudan University Shanghai Cancer Center, Shanghai 200032, China;

${ }^{2}$ Department of Oncology, Shanghai Medical College, Fudan University,

Shanghai 200032, China

*These authors contributed equally to this work
Purpose: Sorafenib is the first-line treatment for patients with unresectable hepatocellular carcinoma (HCC), and its clinical response rate is only about $10 \%$. In clinical practice, some HCC patients obtain favorable overall survival (OS) to the treatment of sorafenib while some patients do not demonstrate a sensitive response to sorafenib. Therefore, it is valuable to determine the subgroups of patients who respond well as well as poorly to sorafenib. Thus, clinical variables of advanced HCC patients with sorafenib treatment were compiled to investigate whether monocyte-to-lymphocyte ratio (MLR) could be a biomarker for predicting sorafenib response.

Patients and methods: In this study, a total of 142 patients with advanced HCC were enrolled from January 1, 2013 to December 31, 2016 at the Fudan University Shanghai Cancer Center. MLR was analyzed using a ROC curve. A Cox regression model and log-rank test were performed to analyze the relationship between clinical factors and OS, as well as progression free survival (PFS).

Results: The optimal cut-off point for MLR was 0.35, and MLR level had no significant correlation with age, gender, hepatitis B infection, grade, alpha-fetoprotein (AFP) level and state of portal vein tumor thrombus. Multivariate Cox regression model showed that grade (HR: 0.608, 95\% CI: 0.409-0.904, $P=0.014$ ), AFP (HR: 0.445, 95\% CI: 0.307-0.645, $P=0.0001$ ), MLR (HR: $0.445,95 \%$ CI: $0.301-0.658, P=0.0001$ ) and aspartate aminotransferase (AST) (HR: $1.005,95 \%$ CI: $1.001-1.009, P=0.007)$ may serve as independent prognostic predictors for OS, and MLR maintained significant correlation with PFS in HCC patients (HR: 0.457, 95\% CI: 0.308-0.678, $P=0.0001)$. By log-rank test, there was longer PFS and OS in patients with low MLR than in those with high MLR (both $P=0.0001$ ).

Conclusion: MLR can predict sorafenib response and a high MLR is correlated with poor prognosis in patients with advanced HCC.

Keywords: hepatocellular carcinoma, sorafenib, monocyte-to-lymphocyte ratio, prognosis

\section{Introduction}

Liver cancer is the sixth most common cancer worldwide, and the third leading cause of cancer-related deaths. ${ }^{1}$ Hepatocellular carcinoma (HCC) accounts for about $70 \%-90 \%$ of liver cancers ${ }^{2}$ that have been diagnosed at an advanced stage at which curative strategies are no longer applicable. Sorafenib, approved by the US Food and Drug Administration in 2007, is the first-line treatment for patients with unresectable HCC. ${ }^{3}$ However, as a small molecule Raf kinase and vascular endothelial growth factor receptor kinase inhibitor, sorafenib has limited survival benefits with low rates of
Correspondence: Zhiqiang Meng Department of Integrative Oncology, Fudan University Shanghai Cancer Center, 270 DongAn Road, Shanghai 200032, China

Tel +86 2I 64175590

Fax +86 21 64437657

Email mengzhq@yeah.net (c)
hereby accept the Terms. Non-commercial uses of the work are permitted without any further permission from Dove Medical Press Limited, provided the work is properly attributed. For permission hereby accept the Terms. Non-commercial uses of the work are permitted without any further permission from Dove Mediect
for commercial use of this work, please see paragraphs 4.2 and 5 of our Terms (https://www.dovepress.com/terms.php). 
tumor response, probably due to the primary and acquired drug resistance mechanisms. ${ }^{4,5}$ Therefore, exploration of biomarkers to predict sorafenib response is urgently warranted, which would determine subsequent treatment strategies.

Since $70 \%-90 \%$ of HCC develop with a background of chronic liver disease, the pathogenesis of HCC mainly involves inflammation. ${ }^{6}$ Increasing evidence shows that markers related to systemic inflammation, including monocyteto-lymphocyte ratio (MLR), neutrophil-to-lymphocyte ratio (NLR), platelet-to-lymphocyte ratio (PLR) and systemic inflammation response index (SIRI) are closely correlated with poor outcomes in different types of carcinomas. ${ }^{7-11}$ Moreover, reports have shown that different types of systemic inflammation cells play distinct roles in prognosis. ${ }^{12-15}$ For example, a high lymphocyte-to-monocyte ratio (LMR) is associated with a good prognosis for advanced pancreatic cancer patients receiving palliative chemotherapy, ${ }^{16}$ while, a high NLR predicts a poor prognosis for small-cell lung cancer. ${ }^{17}$ However, most studies ${ }^{28,30,40}$ have focused on the relationship between system inflammation cells and survival, which ignored the role of inflammatory markers in local and systematic therapy, especially in targeted therapy.

In the present study, a total of 142 patients with advanced HCC treated with sorafenib were enrolled to determine the optimal factor which correlated with survival by Cox regression model and log-rank test. Our data revealed that MLR had significant correlation with overall survival (OS) and progression free survival (PFS) and patients with a high MLR showed a poor prognosis. Our results suggest that MLR was a promising biomarker for predicting sorafenib response in advanced HCC patients.

\section{Patients and methods}

\section{Patients}

A total of 142 patients from the Department of Integrative Oncology at Fudan University Shanghai Cancer Center between January 1, 2013 to December 31, 2017, enrolled in this retrospective study. Informed written consent was obtained from all individual participants. For some patients, HCC can be diagnosed by noninvasive examinations, including an alpha-fetoprotein (AFP) test and the three phase contrast-enhanced computed tomography (CT) or Magnetic Resonance Imaging (MRI). But an pathological confirmation is always required in our study. All medical records, including age, gender, $\mathrm{HBV}$ infection state, grade, AFP level, blood cell counts, liver function tests and state of portal vein tumor thrombus, were collected with written informed consent of the enrolled patients. Barcelona Clinic
Liver Cancer (BCLC) staging system was used to define grade. State of portal vein tumor thrombus was based on the imaging examinations, including ultrasound, computed tomography or MRI.

\section{Treatment and evaluation}

The inclusion criteria for sorafenib treatment: patients aged 18 years and above were deemed eligible for study; patients who had a life expectancy of at least 4 weeks; patients who had an Eastern Co-operative Oncology Group (ECOG) Performance Status of 0 or 1; patients with advanced HCC (unresectable and/or metastatic) which had been histologically documented; patients had to have at least one tumor lesion that could accurately be measured in at least one dimension according to Response Evaluation Criteria in Solid Tumors (RECIST); patients who had not been previously treated with system therapy; patients who had received local therapy, including radiation therapy, and radiofrequency ablation were eligible. Previously treated lesions were not selected as target lesions. Local therapy had to have been completed at least 4 weeks prior to the baseline scan. ${ }^{18}$

A dose of $400 \mathrm{mg}$ sorafenib was orally administered twice a day with or without regular transcatheter arterial chemoembolization therapy. Dose reduction was executed according to the appearance of toxicity. If one of the following situations occurred, a dose adjustment (400 mg once daily) was required until the adverse effects (AEs) were alleviated or eliminated. The situations include gastrointestinal toxicity, hypertension, grade 3/4 hematological toxicity, skin toxicity and hepatic dysfunction defined by the National Cancer Institute's Common Terminology Criteria for Adverse Events (NCI-CTCAE). ${ }^{19}$ If grade 3/4 AEs still existed after dose adjustment, sorafenib treatment was halted until the AEs were alleviated or completely stopped.

Follow-up contrast-enhanced computed tomography or MRI was performed every 8 weeks after sorafenib treatment to assess tumor response and to guide timely decision-making for subsequent therapies. Tumor response was assessed according to the modified RECIST, ${ }^{20,21}$ and were classified into four categories; complete response (CR), partial response (PR), stable disease (SD), and progressive disease (PD). Once PD occurs, other system or local therapies, such as radiation therapy, radiofrequency ablation will have to be adapted. The definition of PFS was calculated from the date of first sorafenib treatment to the date of PD. OS was calculated for all patients from the date of first sorafenib treatment until their death or the last follow-up. 


\section{Statistical analysis}

The aim of using receiver operating characteristic (ROC) curve was to find out the best cut-off value for MLR in order to screen patients who responded well to treatment with sorafenib. The corresponding value at the maximum sensitivity and specificity was defined as the optimal cut-off point. Independent-samples $t$-test was performed to analyze the difference in liver function, age, neutrophil count, monocyte count, lymphocyte count, blood platelet count, NLR, PLR and SIRI with two groups of enrolled patients stratified by MLR. The Chi-squared test was used to analyze two groups with different grade, gender, AFP level and other clinical features. To clarify the prognostic factors of survival, a Cox regression model was applied to identify the best predictor variables using univariate and multivariate analysis. The predictors were estimated with relative risk and $95 \% \mathrm{CI}$ in the regression model. The Log-rank test was used for comparing the OS and
PFS difference between high MLR and low MLR groups. The statistical significance was $P<0.05$ with two-sided. All statistical analyses were performed with SPSS version 19.0 software (IBM Corporation, Armonk, NY, USA).

\section{Ethical approval}

This study was approved by the ethics committee of Fudan University Shanghai Cancer Center. All procedures performed in this study involving human participants were in accordance with the ethical standards of our institutional research committee and with the 1964 Helsinki declaration and its later amendments or comparable ethical standards.

\section{Results}

\section{Baseline patient characteristics}

A total of 142 patients, including 115 males and 27 females, were enrolled in this study. As shown in Table 1, the mean

Table I Association between MLR and clinical characteristics in 142 patients with advanced HCC with sorafenib therapy

\begin{tabular}{|c|c|c|c|c|}
\hline Variables & $\begin{array}{l}\text { Total } \\
(n=142)\end{array}$ & $\begin{array}{l}\text { Low MLR } \\
(\leq 0.35)\end{array}$ & $\begin{array}{l}\text { High MLR } \\
(>0.35)\end{array}$ & $P$-value \\
\hline Age (years) & & & & 0.896 \\
\hline Mean \pm SD & $142(100 \%)$ & $54.29 \pm|.67|$ & $54.00 \pm 1.470$ & \\
\hline Gender & & & & 0.959 \\
\hline Female & 27 (19.1\%) & $13(18.8 \%)$ & 14 (19.2\%) & \\
\hline Male & II 5 (80.9\%) & $56(81.2 \%)$ & $59(81.2 \%)$ & \\
\hline Hepatitis B & & & & 0.421 \\
\hline No & 27 (19.1\%) & 15 (21.7\%) & $12(16.4 \%)$ & \\
\hline Yes & $115(80.9 \%)$ & $54(78.3 \%)$ & $6 \mathrm{I}(83.6 \%)$ & \\
\hline Grade & & & & 0.554 \\
\hline $\mathrm{BCLC} B$ & 46 (32.4\%) & $24(34.8 \%)$ & $22(30.1 \%)$ & \\
\hline BCLC C & $96(67.6 \%)$ & $45(65.2 \%)$ & 51 (69.9\%) & \\
\hline PVTT & & & & 0.363 \\
\hline No & $102(71.8 \%)$ & $52(75.4 \%)$ & $50(68.5 \%)$ & \\
\hline Yes & 40 (28.2\%) & 17 (24.6\%) & $23(31.5 \%)$ & \\
\hline $\operatorname{AFP}(\mathrm{ng} / \mathrm{mL})$ & & & & $0.74 I$ \\
\hline$\leq 400$ & 70 (49.3\%) & $35(50.7 \%)$ & 35 (47.9\%) & \\
\hline$>400$ & $72(50.7 \%)$ & $34(49.3 \%)$ & $38(52.1 \%)$ & \\
\hline Neutrophil count & & & & 0.0523 \\
\hline Mean \pm SD $\left(\times 10^{9} / \mathrm{L}\right)$ & $142(100 \%)$ & $3.422 \pm 1.409$ & $3.929 \pm 1.660$ & \\
\hline Monocyte count & & & & 0.0001 \\
\hline Mean $\pm S D\left(\times 10^{9} / L\right)$ & $142(100 \%)$ & $0.3667 \pm 0.1633$ & $0.4863 \pm 0.1888$ & \\
\hline Lymphocyte count & & & & 0.0001 \\
\hline Mean \pm SD $\left(\times 10^{9} / L\right)$ & $142(100 \%)$ & $1.623 \pm 0.6591$ & $0.9288 \pm 0.3766$ & \\
\hline Platelet count & & & & 0.0026 \\
\hline Mean $\pm \mathrm{SD}\left(\times 10^{9} / \mathrm{L}\right)$ & $142(100 \%)$ & $\mid 80.1 \pm 105.5$ & $134.7 \pm 67.87$ & \\
\hline NLR & & & & $0.04 I I$ \\
\hline Mean \pm SD & $142(100 \%)$ & $11.69 \pm 12.44$ & $8.589 \pm 3.170$ & \\
\hline PLR & & & & 0.03 \\
\hline Mean \pm SD & $142(100 \%)$ & $121.5 \pm 95.38$ & $153.1 \pm 75.98$ & \\
\hline SIRI & & & & 0.0001 \\
\hline$\leq 0.95$ & 56 (39.4\%) & $48(69.6 \%)$ & $8(11 \%)$ & \\
\hline$>0.95$ & $86(60.6 \%)$ & 21 (30.4\%) & 65 (89\%) & \\
\hline
\end{tabular}

Note: Bold values are statistically significant.

Abbreviations: AFP, alpha-fetoprotein; BCLC, Barcelona Clinic Liver Cancer; HCC, hepatocellular cancer; MLR, monocyte-to-lymphocyte ratio; NLR, neutrophil-tolymphocyte ratio; PLR, platelet-to-lymphocyte ratio; PVTT, portal vein tumor thrombus; SIRI, system inflammation response index. 
age at the time of sorafenib treatment was 54.29 years, ranging from 24-86 years. The calculation of SIRI had been reported. ${ }^{41}$ The ROC curve was performed to analyze NLR, MLR, PLR and SIRI to determine the best biomarker and cutoff point (data not show). The AUC of MLR and SIRI were the only two parameters whose value was above 0.6 (0.6918 and 0.6859 respectively) (Figure 1A and B). With the Yourdon's index, the optimal cut-off points of MLR and SIRI were 0.35 and 0.95 . There was no significant difference comparing MLR at baseline and the progression time (Figure 1C). SIRI at baseline showed significant differences with SIRI at the progression time (data not shown).

In consideration of the influence of liver function on sorafenib response, the $t$-test was used to analyze the differences between high MLR and low MLR groups. Results have shown that no differences were found when comparing liver function parameters in two groups (Figure 2).

The patients were divided into two groups according to the cutoff of MLR value for evaluating the association between MLR and clinical characteristics. There were significant differences in the parameters of monocyte count $(P=0.0001)$, lymphocyte count $(P=0.0001)$, platelet count $(P=0.0026)$, $\operatorname{NLR}(P=0.0411), \operatorname{PLR}(P=0.03)$ and SIRI $(P=0.0001)$. In the comparison of other clinical features between two groups, including age, gender, hepatitis B, grade, AFP and PVTT, we found no significant differences (Table 1).

\section{MLR could be a prognostic factor}

As indicated above, MLR and SIRI have the potential to be the serum biomarkers. To make sure that both MRL and
SIRI had the capacity to predict sorafenib responses in HCC patients, survival analysis, including the Cox regression model and log-rank tests were performed. In the univariate Cox regression model, grade, AFP, neutrophil count and MLR were significantly associated with OS $(P=0.007$, $0.0001,0.033$ and 0.007$)$. To avoid the interaction between these parameters, multivariate Cox regression analyses were performed identify the independent factors with OS and PFS. Grade (HR: 0.608, 95\% CI: 0.409-0.904, $P=0.014$ ), AFP (HR: $0.445,95 \%$ CI: $0.307-0.645, P=0.0001)$, MLR (HR: $0.445,95 \%$ CI: $0.301-0.658, P=0.0001)$ and aspartate aminotransferase (AST) (HR: 1.005, 95\% CI: 1.001-1.009, $P=0.007)$ could serve as independent prognostic predictors for OS in HCC patients (Table 2).

To identify the biomarkers for predicting sorafenib response, OS was the first end point event and PFS was the second end point event. The same survival analysis was performed to ascertain the prognostic power of MLR. As shown in Table 3, there was a significant correlation between PFS and grade $(P=0.045), \operatorname{AFP}(P=0.016), \operatorname{MLR}(P=0.019)$ and TBIL $(P=0.031)$ by univariate Cox regression analysis. In a multivariate Cox regression model, MLR was found to be an independent prognostic factor for PFS (HR: 0.457, 95\% CI: $0.308-0.678, P=0.0001)$.

\section{Patients with high MLR had a poor response to sorafenib}

According to the results obtained using the Cox regression model, grade, AFP, MLR and AST had correlations with OS, while only MLR showed a significant correlation with PFS. Therefore, the log-rank test was performed to illustrate
A

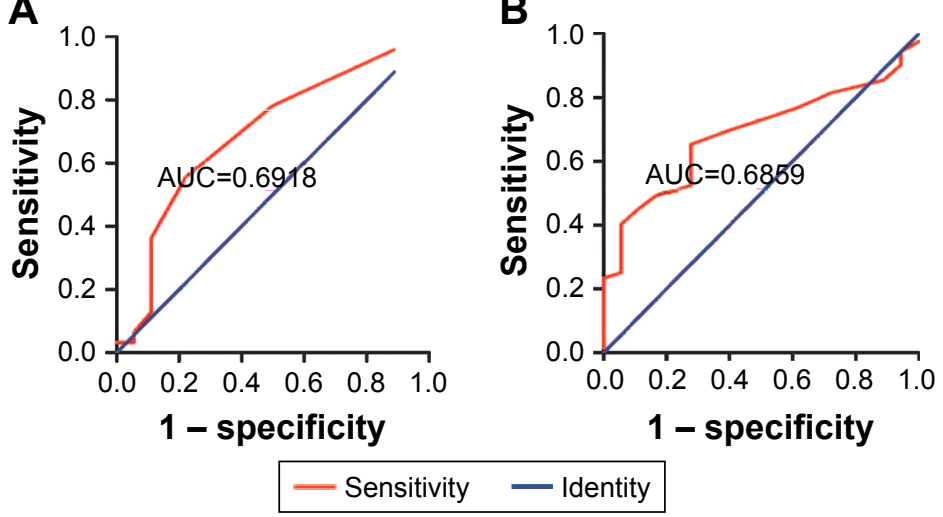

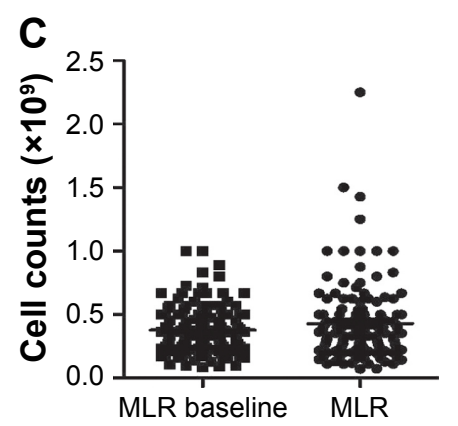

Figure I The ROC curve was performed to test the specificity and sensitivity of MLR and SIRI in HCC patients. MLR and SIRI at baseline, along with the progression time, were analyzed by Independent-samples $t$-test.

Notes: (A) ROC curve of MLR. The area under the curve was 0.6918 . The sensitivity and specificity were $55.6 \%$ and $77.8 \%$, respectively. (B) ROC curve of SIRI. The area under the curve was 0.6859 . The sensitivity and specificity were $65.3 \%$ and $72.2 \%$, respectively. (C) MLR at baseline and the progression time were compared by $t$-test.

Abbreviations: AUC, area under the curve; HCC, hepatocellular cancer; MLR, monocyte to lymphocyte ratio; ROC, receiver operating characteristic; SIRI, systemic inflammation response index. 

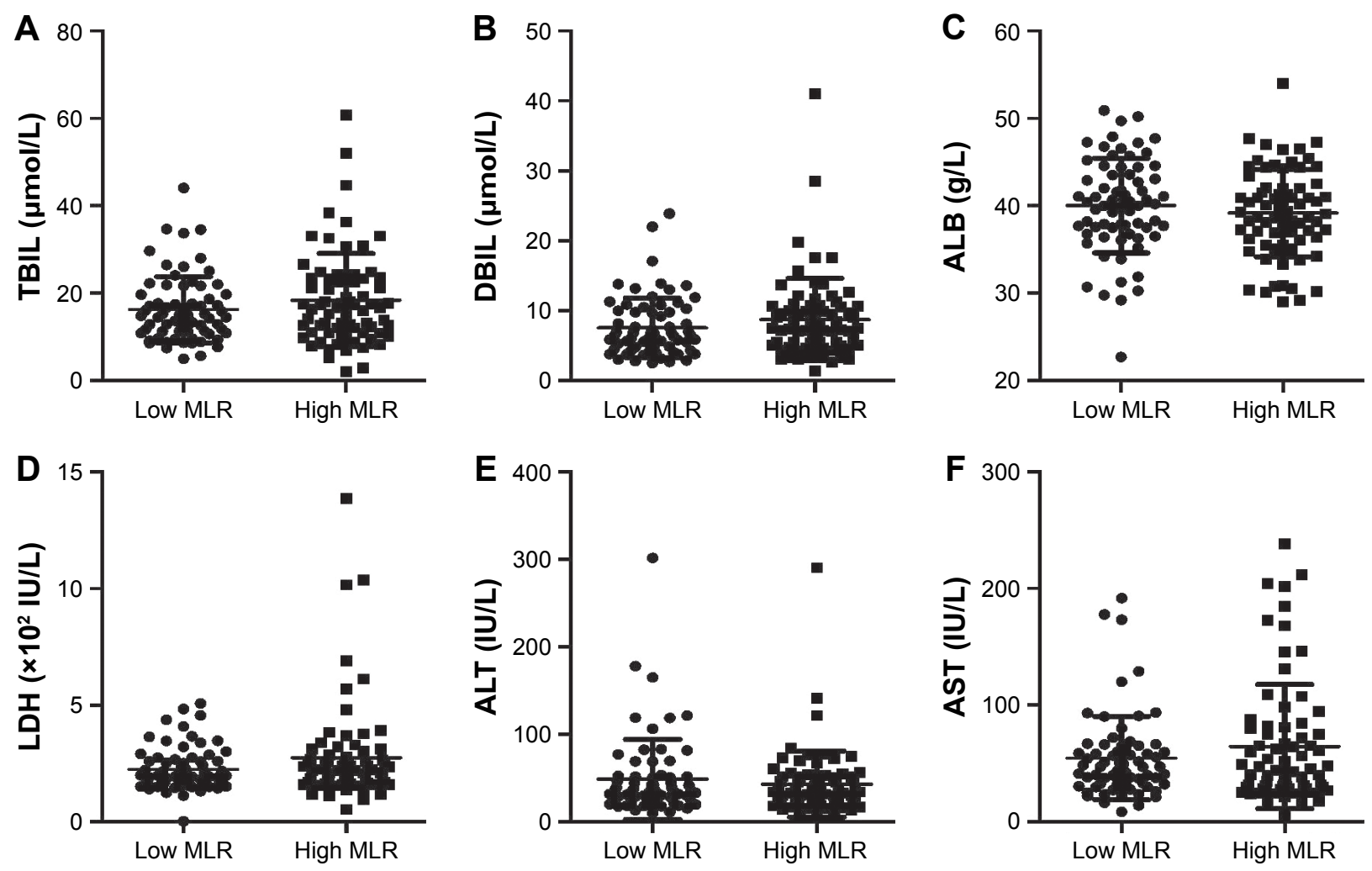

Figure 2 Index parameters of liver function, including TBIL (A), DBIL (B), ALB (C), LDH (D), ALT (E) and AST (F) levels, were analyzed in the low and high MLR groups of patients with HCC by Independent-samples $t$-test.

Note: $P>0.05$.

Abbreviations: ALB, albumin; ALT, alanine aminotransferase; AST, aspartate aminotransferase; DBIL, direct bilirubin; HCC, hepatocellular cancer; LDH, lactic dehydrogenase; MLR, monocyte-to-lymphocyte ratio; TBIL, total bilirubin.

the OS difference between low MLR and high MLR. Meanwhile, the same test was used to analyze different grades, AFP levels and AST levels. As shown in Figure 3A, the median OS was 12.20 months in the BCLC B group, and 9.385 months in the BCLC C group (HR:0.6612, 95\% CI: 0.4581-0.9544). In addition, a significantly longer OS was observed in the BCLC B group than in the BCLC C group. As with the MLR value, the median OS in the low and high MLR groups was 12.90 and 8.20 months respectively (HR: 0.43, 95\% CI: 0.2939-0.6292, Figure 3B). Patients who had a low AFP value had a higher median OS than those with a high AFP value, 14.20 and 7.765 months respectively (HR: 0.4716, 95\% CI: 0.3240-0.6864, Figure 3C). Among the patients who were evaluated for AST levels, the median OS was 12.40 months in the low AST group and 6.50 months in the group with high AST value (HR: 0.5079, 95\% CI: 0.3371-0.7652, Figure 3D).

The difference in PFS between the low MLR and high MLR groups was also tested by log-rank analysis. As shown in Figure 4A, the median progression free survival was 7.0 months in the BCLC B group and 4.6 months in the BCLC C group (HR: 0.7914, 95\% CI: 0.5328-1.176). As with the
MLR value, the median PFS in the low and high MLR groups was 7.9 and 4.0 months respectively (HR: $0.4521,95 \% \mathrm{CI}$ : 0.3030-0.6745, Figure 4B). In addition, significantly longer PFS was observed in the low MLR group than in the high $\operatorname{MLR}$ group $(P=0.0001)$. Among the patients who were evaluated for AFP levels, the median PFS was 7.0 months in the low AFP group and 4.6 months in the group with high AFP value (HR: 0.6697, 95\% CI: 0.4525-0.9912, Figure 4C). Patients who had low AST value had a higher median PFS than those with a high AST value, 6.3 months and 4.85 months, respectively (HR: 0.5882, 95\% CI: 0.3784-0.9142, Figure 4D).

\section{Discussion}

Inflammation is a well-known hallmark of cancer and is substantially attributed to the development and progression of malignancies. ${ }^{22}$ In established cancers, local immune response and systemic inflammation play important roles in disease progression and patient survival. ${ }^{23,24} \mathrm{~A}$ novel inflammatory biomarker, LMR, has been suggested as a promising predicative biomarker for the prognosis of many solid tumors, ${ }^{25-27}$ including $\mathrm{HCC} .{ }^{28-30}$ In our study, the 
Table 2 Univariate and multivariate analysis of overall survival in 142 patients with advanced HCC undergoing sorafenib therapy

\begin{tabular}{|c|c|c|c|c|}
\hline \multirow[t]{2}{*}{ Variables } & \multicolumn{2}{|l|}{ Univariate analysis } & \multicolumn{2}{|c|}{ Multivariate analysis } \\
\hline & HR (95\% Cl) & $P$-value & HR (95\% Cl) & $P$-value \\
\hline Age & $1.006(0.988-1.024)$ & 0.510 & & \\
\hline \multicolumn{5}{|l|}{ Gender } \\
\hline Female/male & $1.003(0.618-1.626)$ & 0.992 & & \\
\hline \multicolumn{5}{|l|}{ Hepatitis B } \\
\hline No/yes & $0.977(0.578-1.652)$ & 0.931 & & \\
\hline \multicolumn{5}{|l|}{ Grade } \\
\hline $\mathrm{BCLC} B / \mathrm{BCLC} C$ & $0.520(0.324-0.835)$ & 0.007 & $0.608(0.409-0.904)$ & 0.014 \\
\hline \multicolumn{5}{|l|}{ PVTT } \\
\hline No/yes & $1.277(0.778-2.096)$ & 0.334 & & \\
\hline \multicolumn{5}{|l|}{ AFP } \\
\hline$\leq 400 \mathrm{ng} / \mathrm{mL} />400 \mathrm{ng} / \mathrm{mL}$ & $0.405(0.266-0.615)$ & 0.0001 & $0.445(0.307-0.645)$ & 0.0001 \\
\hline Neutrophil count & $1.316(1.023-1.693)$ & 0.033 & & \\
\hline Monocyte count & $0.233(0.030-1.826)$ & 0.165 & & \\
\hline Lymphocyte count & $1.028(0.503-2.099)$ & 0.940 & & \\
\hline Platelet count & $1.002(0.996-1.008)$ & 0.473 & & \\
\hline NLR & $1.316(0.926-1.017)$ & 0.212 & & \\
\hline \multicolumn{5}{|l|}{ MLR } \\
\hline$\leq 0.35 />0.35$ & $0.359(0.17 I-0.735)$ & 0.007 & $0.445(0.30 I-0.658)$ & 0.0001 \\
\hline PLR & $0.999(0.993-1.005)$ & 0.707 & & \\
\hline \multicolumn{5}{|l|}{ SIRI } \\
\hline$\leq 0.95 />0.95$ & $0.966(0.548-1.072)$ & 0.904 & & \\
\hline TBIL level & $0.986(0.946-1.028)$ & 0.516 & & \\
\hline DBIL level & $0.999(0.929-1.075)$ & 0.985 & & \\
\hline ALT level & $0.996(0.990-1.002)$ & 0.185 & & \\
\hline AST level & $1.004(0.998-1.010)$ & 0.191 & $1.005(1.001-1.009)$ & 0.007 \\
\hline LDH level & $1.000(0.999-1.002)$ & 0.725 & & \\
\hline ALB level & $0.980(0.937-1.026)$ & 0.393 & & \\
\hline
\end{tabular}

Note: Bold values are statistically significant.

Abbreviations: AFP, alpha-fetoprotein; ALB, albumin; ALT, alanine aminotransferase; AST, aspartate aminotransferase; BCLC, Barcelona Clinic Liver Cancer; DBIL, direct bilirubin; HCC, hepatocellular cancer; LDH, lactic dehydrogenase; MLR, monocyte-to-lymphocyte ratio; NLR, neutrophil-to-lymphocyte ratio; PLR, platelet-to-lymphocyte ratio; PVTT, portal vein tumor thrombus; SIRI, system inflammation response index; TBIL, total bilirubin.

inflammatory biomarkers NLR, MLR, PLR, and SIRI were analyzed by the ROC curve. The AUC of MLR was superior to that of NLR and PLR in the whole study population. The cut-off point of MLR was 0.35 . Univariate analysis revealed that MLR was negatively correlated with OS and PFS of patients with HCC who received sorafenib treatment. In addition, MLR remained an unfavorable prognostic factor for OS and PFS in the multivariate analysis model. Although the cut-off values vary among different types of cancers, our results were consistent with those of previous studies that reported a low pretreatment level of LMR to be an independent unfavorable prognostic factor. ${ }^{28,31}$

Sorafenib could inhibit the proliferation and angiogenesis of tumor cells by targeting the serine threonine kinases Raf-1 and B-Raf and the receptor tyrosine kinase activity of VEGF receptors and PDGF. ${ }^{32}$ Recent studies showed that sorafenib treatment increased the number of $\mathrm{CD} 4^{+} \mathrm{CD} 25^{+}$ regulatory $\mathrm{T}$ cells, ${ }^{33}$ leading to immunosuppression. ${ }^{34,35}$ Circulating monocytes are recruited to the tumor stroma and differentiate into tumor-associated macrophages.
These macrophages are a major component of the tumor microenvironment and can interact with cancer cells to enhance tumor progression by producing various cytokines and chemokines. ${ }^{36,37}$ For the management of patients with advanced HCC, sorafenib is recommended as a category one option for selected patients with Child-Pugh class A liver function and as a category $2 \mathrm{~A}$ option for selected patients with Child-Pugh class B liver function. Clinical trials suggested that the Child-Pugh status is a strong predictor of OS of patients with advanced HCC who are treated with sorafenib. ${ }^{38,39}$ Our results suggested that the median OS and PFS were longer in patients with a low AST level than in those with a high AST level (Figures 3D and 4D). Moreover, our results demonstrated that patients with MLR $>0.35$ had significantly shorter OS and PFS than those with MLR $\leq 0.35$. Thus, MLR and AST have considerable potential as stratification factors in patients with $\mathrm{HCC}$ who are receiving sorafenib treatment.

Despite our important findings, there were some limitations to the present study. Although our hypotheses were 
Table 3 Univariate and multivariate analyses of progression free survival in 142 patients with advanced HCC undergoing sorafenib therapy

\begin{tabular}{|c|c|c|c|c|}
\hline \multirow[t]{2}{*}{ Variables } & \multicolumn{2}{|l|}{ Univariate analysis } & \multicolumn{2}{|c|}{ Multivariate analysis } \\
\hline & HR (95\% CI) & $P$-value & HR (95\% CI) & $P$-value \\
\hline Age & $0.999(0.981-1.016)$ & 0.877 & & \\
\hline \multicolumn{5}{|l|}{ Gender } \\
\hline Female/male & $1.046(0.623-1.763)$ & 0.864 & & \\
\hline \multicolumn{5}{|l|}{ Hepatitis B } \\
\hline No/yes & $1.116(0.648-1.922)$ & 0.693 & & \\
\hline \multicolumn{5}{|l|}{ Grade } \\
\hline $\mathrm{BCLC} B / B C L C C$ & $0.620(0.388-0.990)$ & 0.045 & & \\
\hline \multicolumn{5}{|l|}{ PVTT } \\
\hline No/yes & $1.643(0.970-2.784)$ & 0.065 & & \\
\hline \multicolumn{5}{|l|}{ AFP } \\
\hline$\leq 400 \mathrm{ng} / \mathrm{mL} />400 \mathrm{ng} / \mathrm{mL}$ & $0.595(0.389-0.908)$ & 0.016 & & \\
\hline Neutrophil count & I.I 77 (0.93I-I.489) & 0.173 & & \\
\hline Monocyte count & $0.401(0.052-3.073)$ & 0.379 & & \\
\hline Lymphocyte count & $1.526(0.786-2.960)$ & 0.212 & & \\
\hline Platelet count & $0.998(0.992-1.004)$ & 0.589 & & \\
\hline NLR & $0.996(0.960-1.034)$ & 0.843 & & \\
\hline \multicolumn{5}{|l|}{ MLR } \\
\hline$\leq 0.35 />0.35$ & $0.394(0.181-0.857)$ & 0.019 & $0.457(0.308-0.678)$ & 0.0001 \\
\hline PLR & 1.001 (0.995-1.007) & 0.848 & & \\
\hline \multicolumn{5}{|l|}{ SIRI } \\
\hline$\leq 0.95 />0.95$ & $0.776(0.4|8-| .44 I)$ & 0.422 & & \\
\hline TBIL level & $0.949(0.905-0.995)$ & 0.031 & & \\
\hline DBIL level & $1.073(0.991-1.161)$ & 0.081 & & \\
\hline ALT level & $0.998(0.993-1.004)$ & 0.612 & & \\
\hline AST level & $1.002(0.996-1.008)$ & 0.509 & & \\
\hline LDH level & $1.000(0.999-1.002)$ & 0.695 & & \\
\hline ALB level & $1.001(0.955-1.049)$ & 0.971 & & \\
\hline
\end{tabular}

Note: Bold values are statistically significant.

Abbreviations: AFP, alpha-fetoprotein; ALB, albumin; ALT, alanine aminotransferase; AST, aspartate aminotransferase; BCLC, Barcelona Clinic Liver Cancer; DBIL, direct bilirubin; HCC, hepatocellular cancer; LDH, lactic dehydrogenase; MLR, monocyte-to-lymphocyte ratio; NLR, neutrophil-to-lymphocyte ratio; PLR, platelet-to-lymphocyte ratio; PVTT, portal vein tumor thrombus; SIRI, system inflammation response index; TBIL, total bilirubin.

supported statistically, the cases were collected from one treatment center. Future studies should, therefore, include patients from multiple centers to evaluate whether MLR is retained to predict sorafenib response and correlate with prognosis.

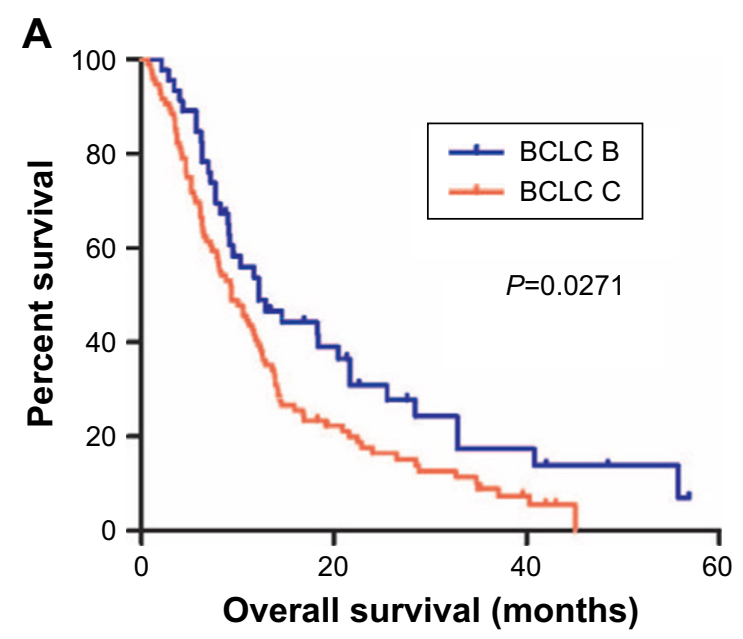

\section{Conclusion}

MLR can predict sorafenib responses in patients with advanced HCC and a relatively high MLR is correlated with a poor prognosis.

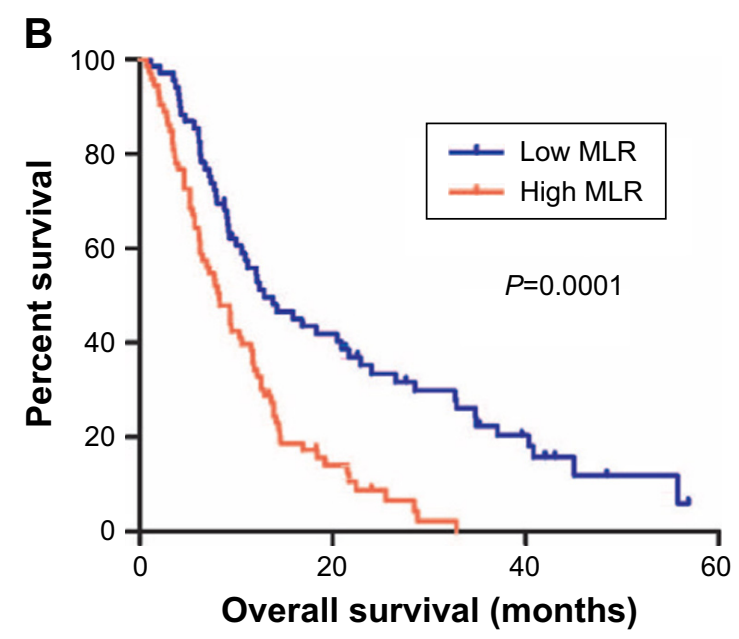

Figure 3 (Continued) 

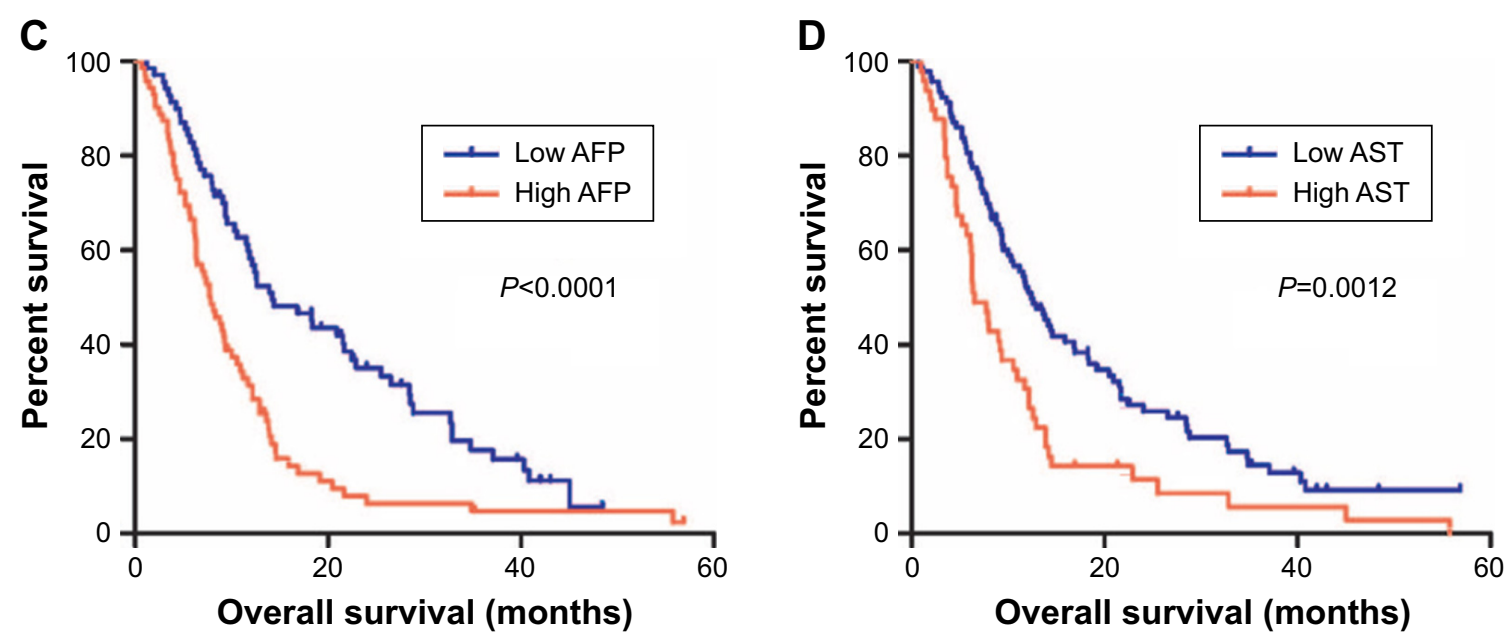

Figure 3 Log-rank tests were used to compare OS in different groups of HCC patients receiving sorafenib therapy, including grade (A), MLR levels (B), AFP levels (C) and AST levels (D).

Abbreviations: AFP, alpha-fetoprotein; AST, aspartate aminotransferase; BCLC, Barcelona Clinic Liver Cancer; HCC, hepatocellular cancer; OS, overall survival; MLR, monocyte-to-lymphocyte ratio.
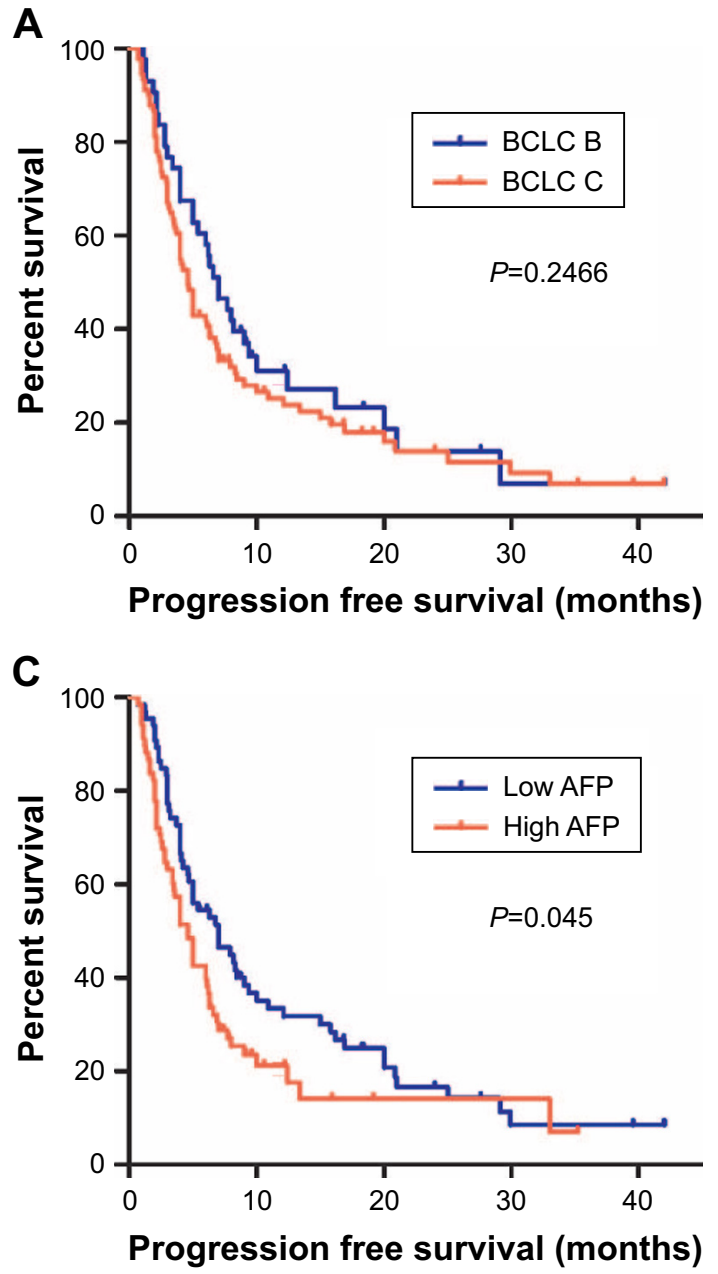
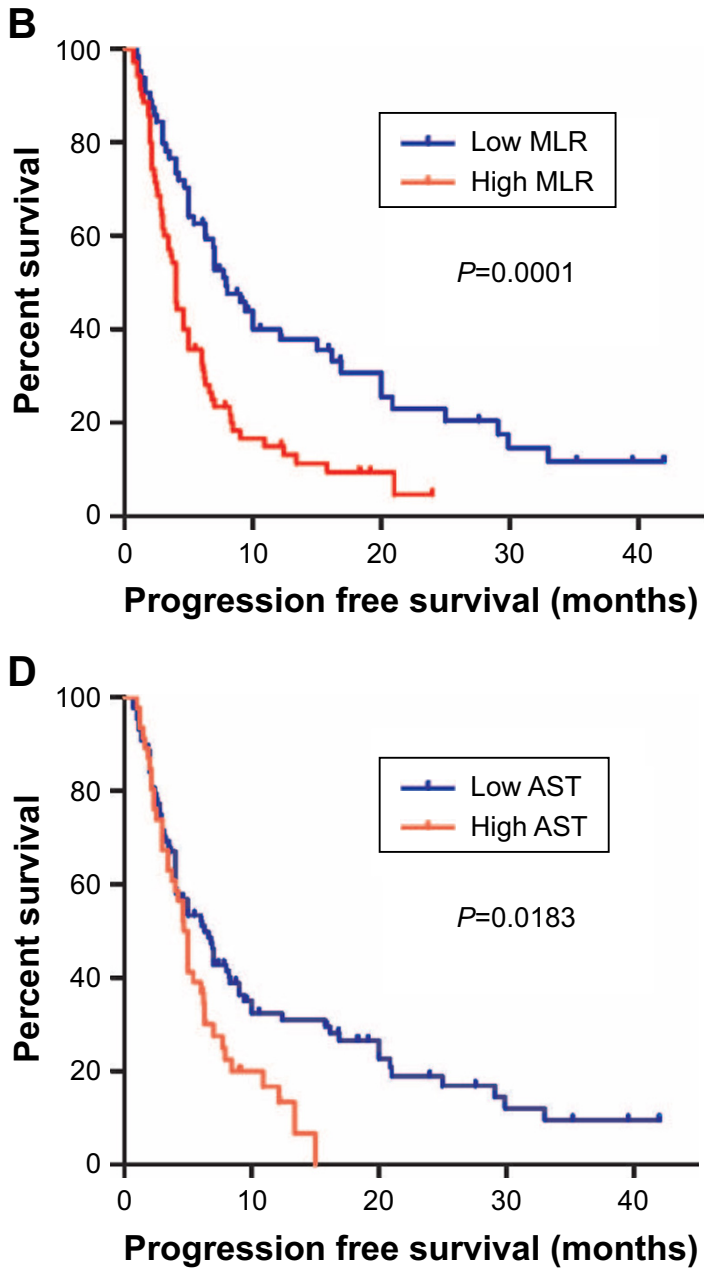

Figure 4 Progression free survival was compared between two groups of patients with advanced HCC receiving sorafenib therapy. Notes: Patients with BCLC C (A), higher MLR (B), higher AFP (C) and higher AST (D) show significantly shorter PFS.

Abbreviations: AFP, alpha-fetoprotein; AST, aspartate aminotransferase; BCLC, Barcelona Clinic Liver Cancer; HCC, hepatocellular cancer; MLR, monocyte-to-lymphocyte ratio; PFS, progression free survival. 


\section{Acknowledgment}

This study was funded by the National Natural Science Foundation of China (grant number 81573753 and 8150140303).

\section{Disclosure}

The authors report no conflicts of interest in this work.

\section{References}

1. Torre LA, Bray F, Siegel RL, Ferlay J, Lortet-Tieulent J, Jemal A. Global cancer statistics, 2012. CA Cancer J Clin. 2015;65(2):87-108.

2. Bruix J, Reig M, Sherman M. Evidence-Based Diagnosis, Staging, and Treatment of Patients With Hepatocellular Carcinoma. Gastroenterology. 2016;150(4):835-853.

3. Llovet JM, Ricci S, Mazzaferro V, et al. Sorafenib in advanced hepatocellular carcinoma. N Engl J Med. 2008;359(4):378-390.

4. Wörns MA, Galle PR. HCC therapies - lessons learned. Nat Rev Gastroenterol Hepatol. 2014;11(7):447-452.

5. Berasain C. Hepatocellular carcinoma and sorafenib: too many resistance mechanisms? Gut. 2013;62(12):1674-1675.

6. Schütte K, Bornschein J, Malfertheiner P. Hepatocellular carcinoma epidemiological trends and risk factors. Dig Dis. 2009;27(2):80-92.

7. Geng Y, Qi Q, Sun M, Chen H, Wang P, Chen Z. Prognostic nutritional index predicts survival and correlates with systemic inflammatory response in advanced pancreatic cancer. Eur J Surg Oncol. 2015; 41(11):1508-1514.

8. Pinato DJ, Stebbing J, Ishizuka M, et al. A novel and validated prognostic index in hepatocellular carcinoma: the inflammation based index (IBI). J Hepatol. 2012;57(5):1013-1020.

9. Li S, Lan X, Gao H, et al. Systemic Inflammation Response Index (SIRI), cancer stem cells and survival of localised gastric adenocarcinoma after curative resection. J Cancer Res Clin Oncol. 2017;143(12): 2455-2468.

10. Xu L, Yu S, Zhuang L, et al. Systemic inflammation response index (SIRI) predicts prognosis in hepatocellular carcinoma patients. Oncotarget. 2017;8(21):34954-34960.

11. Diem S, Schmid S, Krapf M, et al. Neutrophil-to-Lymphocyte ratio (NLR) and Platelet-to-Lymphocyte ratio (PLR) as prognostic markers in patients with non-small cell lung cancer (NSCLC) treated with nivolumab. Lung Cancer. 2017;111:176-181.

12. Romano A, Parrinello NL, Vetro C, et al. Prognostic meaning of neutrophil to lymphocyte ratio (NLR) and lymphocyte to monocyte ratio (LMR) in newly diagnosed Hodgkin lymphoma patients treated upfront with a PET-2 based strategy. Ann Hematol. 2018;97(6):1009-1018.

13. Chan JC, Chan DL, Diakos CI, et al. The Lymphocyte-to-Monocyte Ratio is a Superior Predictor of Overall Survival in Comparison to Established Biomarkers of Resectable Colorectal Cancer. Ann Surg. 2017;265(3):539-546.

14. Dosani T, Covut F, Beck R, Driscoll JJ, de Lima M, Malek E. Significance of the absolute lymphocyte/monocyte ratio as a prognostic immune biomarker in newly diagnosed multiple myeloma. Blood Cancer J. 2017;7(6):e579.

15. Eo WK, Chang HJ, Kwon SH, et al. The Lymphocyte-Monocyte Ratio Predicts Patient Survival and Aggressiveness of Ovarian Cancer. J Cancer. 2016;7(3):289-296.

16. Xue P, Hang J, Huang W, et al. Validation of Lymphocyte-to-Monocyte Ratio as a Prognostic Factor in Advanced Pancreatic Cancer: An East Asian Cohort Study of 2 Countries. Pancreas. 2017;46(8): 1011-1017.

17. Wang X, Teng F, Kong L, Yu J. Pretreatment neutrophil-to-lymphocyte ratio as a survival predictor for small-cell lung cancer. Onco Targets Ther. 2016;9:5761-5770.
18. Won JK, Yu SJ, Hwang CY, et al. Protein disulfide isomerase inhibition synergistically enhances the efficacy of sorafenib for hepatocellular carcinoma. Hepatology. 2017;66(3):855-868.

19. Dueck AC, Mendoza TR, Mitchell SA, et al. Validity and Reliability of the US National Cancer Institute's Patient-Reported Outcomes Version of the Common Terminology Criteria for Adverse Events (PRO-CTCAE). JAMA Oncol. 2015;1(8):1051-1059.

20. Shim JH, Lee HC, Won HJ, et al. Maximum number of target lesions required to measure responses to transarterial chemoembolization using the enhancement criteria in patients with intrahepatic hepatocellular carcinoma. J Hepatol. 2012;56(2):406-411.

21. Kim BK, Kim SU, Kim MJ, et al. Number of target lesions for EASL and modified RECIST to predict survivals in hepatocellular carcinoma treated with chemoembolization. Clin Cancer Res. 2013;19(6): 1503-1511.

22. Hanahan D, Weinberg RA. Hallmarks of cancer: the next generation. Cell. 2011;144(5):646-674.

23. Ishizuka M, Nagata H, Takagi K, Iwasaki Y, Kubota K. Inflammationbased prognostic system predicts survival after surgery for stage IV colorectal cancer. Am J Surg. 2013;205(1):22-28.

24. Hatziveis K, Tourlakis D, Hountis P, et al. Effects on the immune system and toxicity of carboplatin/paclitaxel combination chemotherapy in patients with stage III-IV ovarian and non small cell lung cancer and its role in survival and toxicity. J BUON. 2012;17(1):143-148.

25. Hong YF, Chen ZH, Wei L, et al. Identification of the prognostic value of lymphocyte-to-monocyte ratio in patients with HBV-associated advanced hepatocellular carcinoma. Oncol Lett. 2017;14(2):2089-2096.

26. Zhu JY, Liu CC, Wang L, Zhong M, Tang HL, Wang H. Peripheral blood lymphocyte-to-monocyte ratio as a prognostic factor in advanced epithelial ovarian cancer: a multicenter retrospective study. J Cancer. 2017;8(5):737-743.

27. Yang YT, Jiang JH, Yang HJ, Wu ZJ, Xiao ZM, Xiang BD. The lymphocyte-to-monocyte ratio is a superior predictor of overall survival compared to established biomarkers in HCC patients undergoing liver resection. Sci Rep. 2018;8(1):2535.

28. Li GJ, Ji JJ, Yang F, Xu HW, Bai Y. Preoperative lymphocyte-tomonocyte ratio predicts survival in primary hepatitis B virus-positive hepatocellular carcinoma after curative resection. Onco Targets Ther. 2017;10:1181-1189.

29. Mano Y, Yoshizumi T, Yugawa K, et al. Lymphocyte-to-Monocyte Ratio is a Predictor of Survival after Liver Transplantation for Hepatocellular Carcinoma. Liver Transpl. Epub 2018 Jun 12.

30. Song W, Tian C, Wang K, Zhang RJ, Zou SB. The pretreatment lymphocyte to monocyte ratio predicts clinical outcome for patients with hepatocellular carcinoma: A meta-analysis. Sci Rep. 2017;7:46601.

31. Wu SJ, Lin YX, Ye H, Li FY, Xiong XZ, Cheng NS. Lymphocyte to monocyte ratio and prognostic nutritional index predict survival outcomes of hepatitis B virus-associated hepatocellular carcinoma patients after curative hepatectomy. J Surg Oncol. 2016;114(2): 202-210.

32. Bruix J, Han KH, Gores G, Llovet JM, Mazzaferro V. Liver cancer: Approaching a personalized care. J Hepatol. 2015;62(1 Suppl): S144-S156.

33. Cabrera R, Ararat M, Xu Y, et al. Immune modulation of effector $\mathrm{CD} 4+$ and regulatory $\mathrm{T}$ cell function by sorafenib in patients with hepatocellular carcinoma. Cancer Immunol Immunother. 2013;62(4): 737-746.

34. Flecken T, Schmidt N, Hild S, et al. Immunodominance and functional alterations of tumor-associated antigen-specific CD8+ T-cell responses in hepatocellular carcinoma. Hepatology. 2014;59(4):1415-1426.

35. Sennino B, McDonald DM. Controlling escape from angiogenesis inhibitors. Nat Rev Cancer. 2012;12(10):699-709.

36. Mano Y, Aishima S, Fujita N, et al. Tumor-associated macrophage promotes tumor progression via STAT3 signaling in hepatocellular carcinoma. Pathobiology. 2013;80(3):146-154. 
37. Laoui D, Van Overmeire E, De Baetselier P, Van Ginderachter JA, Raes G. Functional Relationship between Tumor-Associated Macrophages and Macrophage Colony-Stimulating Factor as Contributors to Cancer Progression. Front Immunol. 2014;5:489.

38. Abou-Alfa GK, Amadori D, Santoro A, et al. Safety and Efficacy of Sorafenib in Patients with Hepatocellular Carcinoma (HCC) and Child-Pugh A versus B Cirrhosis. Gastrointest Cancer Res. 2011;4(2): 40-44.

39. Chiu J, Tang YF, Yao TJ, et al. The use of single-agent sorafenib in the treatment of advanced hepatocellular carcinoma patients with underlying Child-Pugh B liver cirrhosis: a retrospective analysis of efficacy, safety, and survival benefits. Cancer. 2012;118(21):5293-5301.
40. Lin ZX, Ruan DY, Li Y, et al. Lymphocyte-to-monocyte ratio predicts survival of patients with hepatocellular carcinoma after curative resection. World journal of gastroenterology. 2015;21(38):10898-10906.

41. Qi Q, Zhuang L, Shen Y, et al. A novel systemic inflammation response index (SIRI) for predicting the survival of patients with pancreatic cancer after chemotherapy. Cancer. 2016;122(14):2158-2167.

\section{Publish your work in this journal}

OncoTargets and Therapy is an international, peer-reviewed, open access journal focusing on the pathological basis of all cancers, potential targets for therapy and treatment protocols employed to improve the management of cancer patients. The journal also focuses on the impact of management programs and new therapeutic agents and protocols on

\section{Dovepress}

patient perspectives such as quality of life, adherence and satisfaction. The manuscript management system is completely online and includes a very quick and fair peer-review system, which is all easy to use. Visit http://www.dovepress.com/testimonials.php to read real quotes from published authors. 